KIAS-P06009

hep-th/0603179

\title{
Supertubes in Field Theories
}

\author{
Seok Kim, Ki-Myeong Lee, Ho-Ung Yee \\ Korea Institute for Advanced Study, Seoul 130-722, Korea \\ e-mail: seok@kias.re.kr, klee@kias.re.kr, ho-ung.yee@kias.re.kr
}

\begin{abstract}
To a domain wall or string object, Noether charge and topological spatial objects can be attracted, forming a composite BPS (Bogomolny-Prasad-Sommerfield) object. We consider two field theories and derive a new BPS bound on composite linear solitons involving multiple charges. Among the BPS objects 'supertubes' appear when the wall or string tension is canceled by the bound energy, and could take an arbitrary closed curve. In our theories, supertubes manifest as Chern-Simons solitons, dyonic instantons, charged semi-local vortices, and dyonic instantons on vortex flux sheet.
\end{abstract}


In last few years there has been some interest in supertubes, which are BPS objects of tubular shape with cross section of arbitrary shape. Initially supertubes as the bound state of D2, D0 branes and fundamental strings (F1) have been found by studying the Dirac-Born-Infeld (DBI) action of D2 branes [1]. Later they have been found in many other context 2, 3, 4, 5. The DBI description of static D2 branes of tubular shape with uniform magnetic flux and electric flux along tube direction shows that the D2 brane tension could be canceled by the bound energy of flux and charge, leading to the energy to be the sum of those for D0 and F1 branes. There would be a linear momentum of constant magnitude flows around the tube, which could lead to nonzero angular momentum.

In this work we ask whether such description suggested by the DBI action analysis of D2 brane is possible in field theories. In a couple of the theories considered here, we find that straight linear topological structures attract both Noether charges and topological spatial solitons and form composite linear BPS objects, with a linear momentum along the line. Among them, there are supertubes, in which the bound energy cancels the tension of the linear structure. Supertubes remain BPS when the linear structure gets bended. In these theories, supertubes manifest as Chern-Simons solitons, dyonic instantons, charged semi-local strings, and dyonic instantons on vortex sheet at Higgs phase, and some more complicated composite configurations.

In field theory, composite BPS objects usually involve only two charges. BPS dyons typify one kind with the energy $\sqrt{\mathcal{Q}^{2}+\mathcal{T}^{2}}$, where $\mathcal{Q}$ and $\mathcal{T}$ are the energies for two charges. Clearly the charge is attractive to magnetic monopoles. Another type is typified by q-lumps whose BPS energy is $|\mathcal{Q}|+|\mathcal{F}|$ where $\mathcal{Q}$ is the energy for the Noether charge and $\mathcal{F}$ is that for the lump [6]. Supertubes in field theories are the generalization of qlumps, and in addition, the interior of supertubes have closed loops of domain wall or string in arbitrary shape. Dyonic instantons and generalizations have been studied as field theoretic supertubes [7, 8].

The composite linear BPS objects may have the energy contributions from five sources. The original linear BPS structure has the tension $\mathcal{T}$. Noether charge leads to the energy density $\mathcal{Q}$, and the spatial topological charge does to $\mathcal{F}$. There is an induced linear momentum density $\mathcal{P}$ along the line, which is not quite independent of $\mathcal{Q}$ and $\mathcal{F}$. In addition the linear structure may be imbedded in additional structure which cost energy density $\mathcal{E}_{0}$. We find the BPS energy per unit length to be

$$
\mathcal{E}_{\text {line }}=\mathcal{E}_{0}+\sqrt{(\mathcal{F} \pm \mathcal{Q})^{2}+(\mathcal{T} \mp \mathcal{P})^{2}} .
$$

This shows what the energy of the composite BPS object is made of. For the supertube 
case, $|\mathcal{T}|=|\mathcal{P}|$, the BPS energy density becomes $\mathcal{E}_{\text {tube }}=\mathcal{E}_{0}+|\mathcal{F}|+|\mathcal{Q}|$, indicating that the tension of the linear structure does not matter anymore. All cases the diagonal component of the energy momentum tension along the momentum direction vanishes, which is another indication that it does not cost any energy to bend the linear structure. The linear momentum density $\mathcal{P}$ is given by the original tension $\mathcal{T}$, and so have constant magnitude. For supertubes of linear shape, one can have $\mathcal{F}$ and $\mathcal{Q}$ arbitrary such that $\mathcal{F} \mathcal{Q}$ is fixed. (If $|\mathcal{F}|=|\mathcal{Q}|$, the BPS configurations are chiral waves along the linear structure.)

Let us start with an abelian Chern-Simons Higgs theory [9, 10, 11, whose Lagrangian is

$$
\mathcal{L}=\frac{\kappa}{2} \epsilon^{\mu \nu \rho} A_{\mu} \partial_{\nu} A_{\rho}-\frac{1}{2}\left|D_{\mu} \phi\right|^{2}-\frac{1}{8 \kappa^{2}}|\phi|^{2}\left(|\phi|^{2}-v^{2}\right)^{2},
$$

where $\epsilon^{012}=1$ and $D_{\mu} \phi=\left(\partial_{\mu}+i A_{\mu}\right) \phi$. There are two degenerated vacua, the symmetric phase $\phi=0$ and the asymmetric phase $\phi=v$, which allow BPS domain walls. There are Chern-Simons solitons in both symmetric and broken phases. The Gauss law is $\kappa F_{12}+\frac{i}{2}\left(D_{0} \phi^{*} \phi-\phi^{*} D_{0} \phi\right)=0$. The Chern-Simons solitons are charge-magnetic flux composite objects, carrying fractional spin and satisfying fractional statistics.

For any field configuration, we rewrite the energy per unit length along a chosen direction, say $x^{2}$, as

$$
\begin{aligned}
\mathcal{E}= & \frac{1}{2} \int d x^{1}\left\{\left|D_{0} \phi-\frac{i}{2 \kappa} \phi\left(|\phi|^{2}-v^{2}\right) \cos \alpha+D_{2} \phi \sin \alpha\right|^{2}\right. \\
& \left.+\left|D_{1} \phi-i D_{2} \phi \cos \alpha+\frac{1}{2 \kappa} \phi\left(|\phi|^{2}-v^{2}\right) \sin \alpha\right|^{2}\right\} \\
& +\mathcal{B} \cos \alpha+(\mathcal{T}-\mathcal{P}) \sin \alpha,
\end{aligned}
$$

where the magnetic flux energy density per unit length, the domain wall energy density, and the linear momentum along the domain wall are, respectively,

$$
\begin{aligned}
\mathcal{B} & =\frac{v^{2}}{2} \int d x^{1} F_{12}, \quad \mathcal{T}=\frac{1}{8 \kappa} \int d x^{1} \partial_{1}\left(2 v^{2}|\phi|^{2}-|\phi|^{4}\right), \\
\mathcal{P} & =\frac{1}{2} \int d x^{1}\left(D_{0} \phi^{*} D_{2} \phi+D_{2} \phi^{*} D_{0} \phi\right) .
\end{aligned}
$$

As all terms in the integration of Eq.(3) is nonnegetive for any angle $\alpha$, we have the bound on the energy density

$$
\mathcal{E} \geq \mathcal{E}_{\text {line }} \equiv \sqrt{\mathcal{B}^{2}+(\mathcal{T}-\mathcal{P})^{2}} \geq \mathcal{E}_{\text {tube }} \equiv|\mathcal{B}|
$$

Note that in the Chern-Simons theory, the Gauss law relates the Noether charge with topological magnetic flux. The composite of domain wall and solitons have been studied before but without the above result [12]. 
Among this linear BPS configurations which saturate the bound $\mathcal{E}=\mathcal{E}_{\text {line }}$, the special ones are those satisfy $\mathcal{T}=\mathcal{P}$, so that $\cos \alpha= \pm 1$. The energy would be determined purely by the magnetic flux as $\mathcal{E}_{\text {tube }}=|\mathcal{B}|$. Thus the wall energy is canceled by the bound energy of the wall and charge, and so the domain wall can bend. This supertube configurations satisfy the Gauss law and the self-dual Chern-Simons soliton equation,

$$
D_{0} \phi \mp \frac{i}{2} \phi\left(|\phi|^{2}-v^{2}\right)=0, \quad D_{1} \phi \mp i D_{2} \phi=0,
$$

which is the equation studied well in Ref. [9, 11, 10]. Thus, if we imagine the effective string action for the supertube, it cost no energy to bend the domain wall. To find explicitly the linear configuration of the supertube, we choose $\cos \alpha=1$ and $\kappa>0$ with ansatz $\phi=|\phi|\left(x^{1}\right) e^{-i v^{2}\left(x^{0}+x^{2}\right) / 2 \kappa}, A_{2}\left(x^{1}\right)=A_{0}\left(x^{1}\right)$. The self-dual equations (6) and the Gauss law can be solved with noting $A_{0}=|\phi|^{2} / 2 \kappa$. The solution is given by $|\phi|^{2}\left(x^{1}\right)=v^{2} e^{v^{2} x^{1} / 2 \kappa}\left(1+e^{v^{2} x^{1} / 2 \kappa}\right)$ with the boundary condition $\phi=0$ at $x=-\infty$ and $|\phi|=v$ at $x=\infty$. From this we can find $\mathcal{T}=\mathcal{P}=v^{4} /(8 \kappa)$ and $\mathcal{F}=v^{4} /(4 \kappa)$. Note that the direction of the momentum flow is fixed by the orientation of the domain walls. This is the case for the Chern-Simons solitons.

While many properties of Chern-Simons solitons are known, we obtain somewhat new perspective of these objects from the fact that they can be regarded as supertubes. (The tubular direction is unclear in this theory at the moment.) In large magnetic flux limit, vortices can be regarded as a collection of supertubes inside of which is in the symmetric phase and outside of which is in the asymmetric phase. Nontopological solitons in large charge limit can be regarded as a collection of supertubes inside of which is in the asymmetric phase and outside of which is in the symmetric phase. One can have nontopological solitons with vortices, which can be regarded as supertubes in supertubes. Almost linear structure of the large flux limit suggests more. For example, the maximal angular momentum can be achieved by a single supertube of circular shape. Indeed, there is such a bound on angular momentum on Chern-Simons solitons [10]. The supersymmetry of these solitons has been studied in $\mathcal{N}=2$ Chern-Simons theory and shown to be $1 / 2$.

The second theory we consider here is a $\mathcal{N}=1 \mathrm{U}(\mathrm{N})$ gauge theory with $N_{f}=0$ or $N_{f} \geq N$ flavors in 5-dim spacetime. For simplicity, we consider the bosonic fields, which are made of the gauge multiplet $A_{M}, A_{5}=\phi$ and the flavor multiplets $q_{f}, q_{f}^{\prime}, f=1 . . N_{f}$. Here $q_{f}^{\prime}$ vanish for the BPS configurations and so are neglected. The bosonic part of the Lagrangian is

$$
\mathcal{L}=\frac{1}{2 e^{2}} \operatorname{tr}\left(-\frac{1}{2} F_{M N} F^{M N}+D_{M} \phi D^{M} \phi-D^{2}\right)
$$




$$
+\frac{1}{2} \operatorname{tr}\left(D_{M} \bar{q}_{f} D^{M} q_{f}-\left(\phi-m_{f}\right)^{2} \bar{q}_{f} q_{f}\right)
$$

where the nonvanishing D-term is $D=D^{3}=e^{2}\left(v^{2}-\bar{q}_{f} q_{f}\right) / 2$ and $D_{M} q_{f}=\partial_{M} q_{f}+i q_{f} A_{M}$. The Gauss law is

$$
D_{\mu} F_{\mu 0}+i\left[\phi, D_{0} \phi\right]+\frac{i e^{2}}{2}\left(\bar{q}_{f} D_{0} q_{i}-D_{0} \bar{q}_{f} q_{f}\right)=0
$$

The supersymmetry transformation of the guagino field $\lambda_{i}$ and the matter fermion $\psi_{f}$ is given as (see, for example [13, 14] .)

$$
\delta \lambda_{i}=\frac{1}{2} F_{M N} \Gamma^{M N} \epsilon_{i}+i \mathbf{D}^{a} \sigma_{i j}^{a} \epsilon_{j}, \delta \psi_{f}=D_{M} q_{f i} \Gamma^{M} \epsilon_{i}
$$

in 6-dim notation with symplectic Majorana-Weyl spinor $\epsilon_{i}, \lambda_{i}$. For our theory, $q_{f 1}=$ $q_{f}, q_{f 2}=q_{f}^{\prime}$ and $D_{5} q_{f}=q_{f}\left(\phi-m_{f}\right)$. We impose an $1 / 4$ BPS condition on the spinor parameter by imposing two conditions,

$$
\Gamma^{34} e^{\alpha \Gamma^{45}} i \sigma_{i j}^{3} \epsilon_{j}=\epsilon_{i}, \quad \Gamma^{05} e^{\alpha \Gamma^{45}} \epsilon_{i}= \pm \epsilon_{i},
$$

which implies $\Gamma^{12} i \sigma_{i j}^{3} \epsilon_{j}=\mp \epsilon_{i}$ as $\epsilon_{i}$ is chiral. For such constant nonzero spinor $\epsilon_{i}$, the supersymmetric transformations $\delta \lambda_{i}$ and $\delta \psi_{f}$ vanish if the bosonic configurations satisfy the BPS equations

$$
\begin{aligned}
& B_{i} \pm \delta_{i 3} D^{3} \mp F_{i 4} \cos \alpha \mp D_{i} \phi \sin \alpha=0, \\
& F_{i 0} \pm F_{i 4} \sin \alpha \mp D_{i} \phi \cos \alpha=0, \\
& F_{40} \mp D_{4} \phi \cos \alpha=0, D_{0} \phi \pm D_{4} \phi \sin \phi=0, \\
& \left(D_{1} \pm i D_{2}\right) q_{f}=0, \\
& D_{0} q_{f} \pm D_{4} q_{f} \sin \alpha \mp i q_{f}\left(\phi-m_{f}\right) \cos \alpha=0, \\
& D_{3} q_{f}-i D_{4} q_{f} \cos \alpha+q_{f}\left(\phi-m_{f}\right) \sin \alpha=0,
\end{aligned}
$$

where $B_{3}=F_{12}$ and so on. The energy density is given by the square of the above terms with proper coefficient and the boundary terms after the Gauss law is used. As the square terms are positive definite, we get a bound on the energy density

$$
\mathcal{E} \geq \mp \frac{v^{2}}{2} \operatorname{tr} F_{12}+(\mathcal{F} \pm \mathcal{Q}) \cos \alpha+(\mathcal{T} \mp \mathcal{P}) \sin \alpha
$$

where $\alpha$ is arbitrary and

$$
\begin{aligned}
\mathcal{F} & =\frac{v^{2}}{2} \operatorname{tr} F_{34} \pm \frac{1}{e^{2}} \operatorname{tr} B_{i} F_{i 4}, \\
\mathcal{Q} & =\frac{1}{e^{2}} \partial_{\mu} \operatorname{tr}\left(F_{\mu 0} \phi\right)+\frac{i}{2} m_{f} \operatorname{tr}\left(\bar{q}_{f} D_{0} q_{f}-D_{0} \bar{q}_{f} q_{f}\right),
\end{aligned}
$$




$$
\begin{aligned}
\mathcal{T}= & \pm \frac{1}{e^{2}} \partial_{i} \operatorname{tr}\left(B_{i} \phi\right)+\frac{1}{2} \partial_{3} \operatorname{tr}\left(v^{2} \phi-\bar{q}_{f} q_{f}\left(\phi-m_{f}\right)\right), \\
\mathcal{P}= & \frac{1}{e^{2}} \operatorname{tr}\left(F_{i 0} F_{i 4}+D_{0} \phi D_{4} \phi\right) \\
& +\frac{1}{2} \operatorname{tr}\left(D_{0} \bar{q}_{f} D_{4} q_{f}+D_{4} \bar{q}_{f} D_{0} q_{f}\right) .
\end{aligned}
$$

The first term $F_{12}$ term comes from the magnetic flux vortex sheet. The linear structure of magnetic monopole string or domain wall lead to the tension part $\mathcal{T}$. The linear momentum density along $x^{4}$ is $\mathcal{P}$. $\mathcal{Q}$ is the energy due to the flavor charge. $\mathcal{F}$ is the energy density due to the magnetic flux $\operatorname{tr} F_{34}$ and the instanton density. We have to integrate over the transverse direction to the linear structure to get the energy density, leading to the BPS energy density (10). In a gauge $A_{0}= \pm \eta \phi$, the supertube configurations, which saturate the energy bound with $\cos \alpha=\eta= \pm 1$, satisfy the following equations,

$$
\begin{aligned}
& B_{i} \mp \eta F_{i 4} \pm \delta_{i 3} \frac{e^{2}}{2}\left(v^{2}-\bar{q}_{f} q_{f}\right)=0, \\
& \left(D_{1} \pm i D_{2}\right) q_{f}=0,\left(D_{3}-i \eta D_{4}\right) q_{f}=0 \\
& D_{i}^{2} \phi-\frac{e^{2}}{2}\left(\left(\phi-m_{f}\right) \bar{q}_{f} q_{f}+\bar{q}_{f} q_{f}\left(\phi-m_{f}\right)\right)=0 .
\end{aligned}
$$

Note that the first three equations are for the spatial structure. The last one is additional structure on the spatial structure. Typically the spatial solitons have some scaling parameters, which collapse when some symmetry is further broken. But the repulsive force among Noether charge balances the collapsing force, making supertubes as dyonic spatial solitons.

We will consider the four supertube examples in this theory. First of all, let us neglect all flavor. The supersymmetry can enlarged to $\mathcal{N}=2$ in 5 -dim if we add an adjoint matter multiplet. Assume the gauge group is $S U(2)$. Then the obvious linear object is the monopole string, to which electric charge and instantons are attracted. The integration all above densities over three dimensional space transverse monopole string leads to the BPS bound (11) on the energy density along the string. (Here we are a bit loose on the notation for the energy densities and its integrated quantities.) Here the 16 susy leads to more sign freedom and so more sign combinations are allowed. When $\cos \alpha= \pm 1$, we get the supertube configuration, whose BPS equation (14) becomes that for dyonic instantons, in which arbitary shaped closed loops of magnetic monopoles are present.

For a single monopole string with known $A_{i}, \phi$, the linear density for these quantities are trivial to get with $\phi=v A_{4} / h$ where $A_{4}=h \sigma^{3} / 2$ and $\phi=v \sigma^{3} / 2$ asymptotically. They are $\mathcal{T}=\mathcal{P}=4 \pi v / e^{2}$ and $\mathcal{F}=4 \pi h / e^{2}$ and $\mathcal{Q}=4 \pi v^{2} / e^{2} h$, and so $\mathcal{Q} \mathcal{F}$ is independent of $h$. This tell us the characterstics of dyonic instantons in large instanton and charge limit, 
where the linear approximation for the monopole strings may work. Dyonic instantons have been studied studied in many directions [15, 16]. The monopole string composite with charge and instantons have been also studied [17, 18]. Monopole strings inside dyonic instantons appear when the instanton number is at least two, showing they are supertubes[7].

Second case is the $U(1)$ theory with $N_{f}=2$. When the flavor symmetry is nonabelian with $m_{1}=m_{2}$, the theory allows semi-local vortices [19, 20]. With $m_{1}<m_{2}$, there are two degenerated vacua with $q_{1}=v, q_{2}=0, \phi=m_{1}$ and $q_{1}=0, q_{2}=v, \phi=m_{2}$, which allows a domain wall [21, 22]. With $m_{1}<m_{2}$, the core of the semi-local vortices collapse. We ignore $x^{1}$ and $x^{2}$ direction completely. The domain wall attracts the flavor charge and magnetic flux $F_{34}$. For energy density along the line $x^{4}$, we integrate over $x^{3}$, with $\mathcal{F}$ due to the magnetic flux $F_{34}$. Again we obtain BPS energy bound (10) per length. Especially for $\cos \alpha= \pm 1$, the domain wall can bend, and the BPS equations (14) becomes those for charged semi-local vortices [24]. For a straight domain wall with ansatz $q_{f}=\left|q_{f}\right| e^{-m_{f} x^{0}-i k_{f} x^{3}}$ with field choice so that $m_{2}=-m_{1}=m>0$ and $k_{2}=-k_{1}=$ $k>0$, with appropriate boundary condition, we see that $A_{4}=k \phi / m$ and so obtain that $\mathcal{T}=\mathcal{P}=m v^{2}$ and $\mathcal{F}=k v^{2}$ and $\mathcal{Q}=m v^{2} / 2 k$. Again $\mathcal{F} \mathcal{Q}$ is independent of $k$ and the component $T_{44}$ along the domain wall direction vanishes.

A supertube for generic charged semi-local vortices of $U(1)$ theory with two flavors of different mass with a large magnetic flux can be caricatured as follows. Say the vacuum is where $q_{1}=v, q_{2}=0$. The vortex lies in 3-4 plane, and we ignore 1,2 direction. For large vorticity, there is an arbitrary shaped closed line of domain wall, outside of which $\left|q_{1}\right|=v$ and $q_{2}=0$, and inside of which $q_{1}=0$ and $\left|q_{2}\right|=v$. Along the line there are energy density which is identical to the momentum density given above. There are nontrivial magnetic flux and flavor charge which can vary along the line as long as their product is fixed as in the previous paragraph.

The third case appears in the $U(2)$ gauge theory with two flavors. The vacuum is in the color-flavor locking phase $\bar{q}_{1}=(v, 0), \bar{q}_{2}=(0, v)$ and $\phi=\operatorname{diga}\left(m_{1}, m_{2}\right)$. We consider a vortex sheet on the 3-4 plane at $x^{1}, x^{2} \sim 0$ with nonzero $F_{12}$, and with a magnetic monopole string along $x^{4}$ direction at $x^{3} \sim 0$ when $m_{1} \neq m_{2}$ [25, 23]. The gauge orientation of the magnetic flux $F_{12}$ changes from $\operatorname{diag}(1,0)$ to $\operatorname{diag}(0,1)$ as $x^{3} \operatorname{goes}$ from $-\infty$ to $\infty$. Thus the energy would have a contribution $\mathcal{E}_{0}$ from the magnetic flux $\operatorname{tr} F_{12}$ which is localized near $x^{1}=x^{2}=0$ and the monopole string tension $\mathcal{T}$. Monopole strings attract flavor charge and instantons, which leads to energy contribution $\mathcal{Q}$ and $\mathcal{F}$. Integration over $x^{1}, x^{2}, x^{3}$ with some appropriate infrared cutoff in $x^{3}$ direction would 
lead to a energy density bound (10. The BPS equations (14) becomes that for dyonic instantons on the vortex sheet, where $q_{f} \sim e^{-i m_{f} x^{0}}$. When $m_{1}=m_{2}$, one can have instantons in the vortex sheet in the Higgs phase [26], which collapses $m_{1} \neq m_{2}$. Flavor Noether charges keep them from collapsing.

For the linear configuration with additional ansatz $q_{f} \sim e^{-i k_{f} x^{4}}$, and we shift the fields $A_{4}$ and $\phi$ so that $m_{1}=-m_{2}=m / 2$ and $k_{1}=-k_{2}=k / 2$, which implies asymptotic value of $A_{4}=\operatorname{diag}(k,-k) / 2$, and $\phi=\operatorname{diag}(m,-m) / 2$. The selfdual equation and the gauss law are compatible if $A_{4}=k \phi / m$. For a single monopole string, we obtain $\mathcal{T}=\mathcal{P}=4 \pi m / e^{2}$ and $\mathcal{F}=4 \pi k / e^{2}$ and $\mathcal{Q}=4 \pi m / k$ after some effort. Again $\mathcal{F} \mathcal{Q}$ is independent of $k$.

Then for large instanton number and flavor charge, the supertube configuration can be pictured as follows. We have a flat vortex sheet on which an arbitrary shaped closed curve of monopole string is present. On the sheet, outside of the curve $q_{1}=0, q_{2}=\operatorname{diag}(0, v)$ at the sheet center and inside of the curve $q_{1}=\operatorname{diag}(v, 0)$ and $q_{2}=0$. On the curve, there are nonzero flavor charge and instanton density per unit length, their product remain uniform. (It is of course not well defined unless the curve can be approximated to be straight.) Along the curve, there is a linear momentum flows with unform magnitude given by the monopole tension. This configuration would become dyonic instanton in the Coulomb when the FI parameter $v^{2}$ approaches zero.

For the last case, we consider the theory with $U(2)$ gauge group and $N_{f}=3$ case. As there are degenerated vacua, one can have domain walls. In addition, one can have vortices sheet with magnetic monopoles strings, which allows a composition of vortex-monopoledomain wall [27, 28]. Our BPS equation allows adding instanton numbers, flavor charges and additional flux $F_{34}$. Consequently we can imagine a composition of charged semi-local vortices and an dyonic instanton sin the vortex sheet. The interpretation of the above field theory as the field theory on D4-D8 branes seems to show above supertubes in nice figurative way.

To conclude, we found a new BPS energy bound on straight composite linear BPS objects. Especially supertubes seem to be everywhere when one can add Noether charge to topological solitons. While our analysis has been done on relatively simpler systems, one can generalization to nonabelian gauge groups and more matter fields, which would allow richer supertube structures.

This work is supported in part by KOSEF Grant R01-2003-000-10319-0, KOSEF SRC Program through CQUeST at Sogang (K.L.), and Korean Research Foundation Grant No. KRF-2005-070-C00030 (K.L.). The authors (K.L. and H.U.Y.) thank the Yukawa 
Institute for Theoretical Physics at Kyoto University, where the part of the work was motivated during the YITP-W-05-21 (a workshop) on "Fundamental Problems and Applications of Quantum Field Theory".

\section{References}

[1] D. Mateos and P. K. Townsend, Phys. Rev. Lett. 87, 011602 (2001) arXiv:hep-th/0103030.

[2] D. Bak and K. M. Lee, Phys. Lett. B 509, 168 (2001).

[3] R. Emparan, D. Mateos and P. K. Townsend, JHEP 0107, 011 (2001) arXiv:hep-th/0106012.

[4] D. s. Bak and A. Karch, Nucl. Phys. B 626, 165 (2002).

[5] D. Mateos, S. Ng and P. K. Townsend, JHEP 0203, 016 (2002)

[6] R. A. Leese, Nucl. Phys. B 366, 283 (1991).

[7] S. Kim and K. M. Lee, JHEP 0309, 035 (2003)

[8] P. K. Townsend, Comptes Rendus Physique 6, 271 (2005) arXiv:hep-th/0411206.

[9] J. Hong, Y. Kim and P. Y. Pac, Phys. Rev. Lett. 64, 2230 (1990).

[10] R. Jackiw, K. M. Lee and E. J. Weinberg, Phys. Rev. D 42, 3488 (1990).

[11] R. Jackiw and E. J. Weinberg, Phys. Rev. Lett. 64, 2234 (1990).

[12] H. c. Kao, K. M. Lee and T. Lee, Phys. Rev. D 55, 6447 (1997) arXiv:hep-th/9612183.

[13] K. Lee and H. U. Yee, Phys. Rev. D 72, 065023 (2005) arXiv:hep-th/0506256.

[14] M. Eto, Y. Isozumi, M. Nitta and K. Ohashi, arXiv:hep-th/0506257.

[15] N. D. Lambert and D. Tong, Phys. Lett. B 462, 89 (1999) arXiv:hep-th/9907014.

[16] E. Eyras, P. K. Townsend and M. Zamaklar, JHEP 0105, 046 (2001) arXiv:hep-th/0012016.

[17] D. s. Bak and K. M. Lee, Phys. Lett. B 544, 329 (2002) arXiv:hep-th/0206185. 
[18] K. M. Lee and S. H. Yi, Phys. Rev. D 67, 025012 (2003) arXiv:hep-th/0205274.

[19] T. Vachaspati and A. Achucarro, Phys. Rev. D 44, 3067 (1991).

[20] M. Hindmarsh, Phys. Rev. Lett. 68, 1263 (1992).

[21] D. Tong, Phys. Rev. D 66, 025013 (2002) arXiv:hep-th/0202012.

[22] Y. Isozumi, K. Ohashi and N. Sakai, JHEP 0311, 060 (2003) arXiv:hep-th/0310189.

[23] M. Shifman and A. Yung, Phys. Rev. D 70, 045004 (2004) arXiv:hep-th/0403149.

[24] E. Abraham, Nucl. Phys. B 399, 197 (1993).

[25] D. Tong, Phys. Rev. D 69, 065003 (2004) arXiv:hep-th/0307302.

[26] M. Eto, Y. Isozumi, M. Nitta, K. Ohashi and N. Sakai, Phys. Rev. D 72, 025011 (2005) arXiv:hep-th/0412048.

[27] N. Sakai and D. Tong, JHEP 0503, 019 (2005) arXiv:hep-th/0501207.

[28] R. Auzzi, M. Shifman and A. Yung, Phys. Rev. D 72, 025002 (2005) arXiv:hep-th/0504148. 